\title{
Comunicação
}

\section{Avaliação da qualidade fisiológica de sementes de feijão-caupi pelo teste de condutividade elétrica}

\author{
Nathalia Aparecida Silva Batista ${ }^{1}$, Petterson Baptista da Luz ${ }^{2}$, Severino de Paiva Sobrinho ${ }^{3}$, \\ Leonarda Grillo Neves ${ }^{4}$, Willian Krause ${ }^{2}$
}

\section{RESUMO}

A medição da condutividade elétrica da solução de embebição de sementes é um procedimento recomendado para avaliar o vigor de sementes de ervilha, sugerido para as de soja. Para as de outras plantas, porém, ainda não está bem fundamentada. Este trabalho teve como objetivo avaliar a qualidade fisiológica de sementes de feijão-caupi. Para isso, o estudo foi conduzido, utilizando-se acessos de feijão-caupi, representados por quatro lotes de sementes. Foram realizados os testes de germinação, primeira contagem de germinação, emergência de plântulas e de condutividade elétrica, usando-se a temperatura de $25^{\circ} \mathrm{C}$; volumes de água para embebição de 75 e $100 \mathrm{~mL}$, amostras de 25 e 50 sementes; e períodos de embebição de 2, 4, 8, 12 e 24 horas. Utilizou-se o delineamento experimental inteiramente casualizado, com quatro repetições, avaliando-se os efeitos de lotes. Diante dos resultados obtidos, verificou-se que o teste de condutividade elétrica foi eficiente na separação dos lotes de sementes em diferentes níveis de vigor.

Palavras-chave: Vigna unguiculata (L.) Walp., germinação, vigor, potencial fisiológico.

\begin{abstract}
Evaluation of cowpea seed quality by electrical conductivity

The electrical conductivity of the seed soaking solution is a procedure recommended for the evaluation of vigor in pea and soybean seeds. However, it was not so well established in other species. The objective of this work was to evaluate their effects on physiological quality of cowpea seeds. Four batches of cowpea seeds were tested for germination, first count of germination, germination percentage, emergence percentage, rate of emergence, radicle and variations in electrical conductivity (temperature $25^{\circ} \mathrm{C}$; volumes of $75 \mathrm{~mL}$ and $100 \mathrm{~mL}$ of water, 25 and 50 seeds, and 2 , 4, 8, 12 and 24 hours periods). The experimental design was a completely randomized with four replicates for each treatment. Based on these results, it was found that the electrical conductivity test was efficient in the separation of seed lots according to their vigor levels.
\end{abstract}

Key words: Vigna unguiculata L., germination, seed vigor, physiological potential.

Recebido para publicação em 27/09/2011 e aprovado em 09/08/2012

${ }^{1}$ Graduanda em Agronomia. Universidade do Estado de Mato Grosso, Avenida São João, s/n, Bairro Cavalhada, 78200-000, Cáceres, Mato Grosso, Brasil. nathalia.caceres@yahoo.com.br

${ }^{2}$ Engenheiros-Agrônomos, Doutores. Departamento de Agronomia, Universidade do Estado de Mato Grosso, Avenida São João, s/n, Bairro Cavalhada, 78200-000, Cáceres, Mato Grosso, Brasil. petterbaptista@yahoo.com.br (autor correspondente), krause@ unemat.br

${ }^{3}$ Engenheiro-Agrônomo, Mestre. Departamento de Agronomia, Universidade do Estado de Mato Grosso, Avenida São João, s/n, Bairro Cavalhada, 78200-000, Cáceres, Mato Grosso, Brasil. paivasobrinho@unemat.br

${ }^{4}$ Engenheira Agrônoma, Doutora. Departamento de Agronomia, Universidade do Estado de Mato Grosso, Avenida São João, s/n, Bairro Cavalhada, 78200-000, Cáceres, Mato Grosso, Brasil. leonardaneves@unemat.br 


\section{INTRODUÇÃO}

O feijão-caupi, feijão-de-corda ou feijão-macassar (Vigna unguiculata (L.) Walp.) é uma das mais adaptáveis, versáteis e nutritivas leguminosas cultivadas. É, por isso, muito utilizado como forragem verde, feno, ensilagem, farinha para alimentação animal, adubação verde e proteção do solo (Dutra \& Teófilo, 2007).

A alta qualidade de sementes é de grande importância, principalmente quanto à germinação uniforme, necessária para garantir um estande ideal de plantas. Por essa razão, sementes de alto vigor constituem-se em elemento básico e fundamental da cultura (Mendonça et al., 2003).

Para avaliar a qualidade das sementes, alguns testes vêm sendo recomendados. O teste de germinação tem sido utilizado para determinar a capacidade das sementes de produzirem plântulas normais, sob condições favoráveis de ambiente (Carvalho \& Nakagawa, 2000).

Segundo Carvalho et al. (2006), existem várias limitações para retratar o desempenho do lote em condições de campo. Marcos Filho (1999a) relatou que, quando as condições de ambiente desviam-se das mais adequadas, a avaliação do vigor é necessária para estimar o potencial de desempenho das sementes. Segundo o autor, os testes de vigor devem ser escolhidos de maneira a atender os objetivos específicos, completando as informações obtidas no teste de germinação. De acordo com as Regras de Análise de Sementes (RAS) (Brasil, 2009), considera-se germinada toda a semente que, pelo desenvolvimento das estruturas essenciais de seu embrião, mostre sua capacidade para gerar uma planta normal, sob condições ambientais favoráveis. Porém, ao se realizar o teste, devem-se levar em consideração os fatores edafoclimáticos, pois, no processo de germinação ocorre uma série de atividades metabólicas, baseadas em reações químicas e apresentando exigências quanto à temperatura e à disponibilidade de oxigênio (Marcos Filho, 1986).

Além da necessidade de padronização de métodos e interpretação dos resultados, os testes de vigor devem apresentar relação com a emergência de plântulas em campo, rapidez, objetividade, simplicidade, baixo custo e reprodutibilidade (AOSA, 1983)

O teste de condutividade elétrica é um meio rápido e prático de se determinar o vigor de sementes, podendo ser conduzido facilmente na maioria dos laboratórios de análise de sementes, sem maiores despesas com equipamento e treinamento de pessoal (Vieira \& Krzyzanowski, 1999). Sua avaliação pode ser medida em função da quantidade de íons lixiviados, estando diretamente relacionado com a integridade das membranas celulares. Desta forma, membranas mal estruturadas e células danificadas estão, geralmente, associadas com o processo de deterioração da semente, ou seja, com sementes de baixo vigor (Vieira \& Krzyzanowski,1999; AOSA, 2002).
Objetivou-se, neste trabalho, avaliar a qualidade fisiológica de sementes de feijão-caupi, por meio do teste de condutividade elétrica.

\section{MATERIAL E MÉTODOS}

O trabalho foi realizado no laboratório de Sementes da Universidade do Estado de Mato Grosso - UNEMAT, campus de Cáceres, utilizando-se quatro lotes de sementes de feijão-caupi, do cultivar BRS Guariba. Essas sementes foram produzidas no município de Sinop, durante o ano de 2010, e mantidas em ambientes de câmara fria (5 ${ }^{\circ} \mathrm{C}$ e $40 \%$ UR), acondicionadas em embalagens de papel multifoliado, durante quatro meses. A avaliação da qualidade fisiológica das sementes de feijão-caupi foi determinada por testes de germinação, de emergência e pelo teste de condutividade elétrica.

O grau de umidade foi determinado pelo método da estufa, a $105{ }^{\circ} \mathrm{C}\left(+/-3{ }^{\circ} \mathrm{C}\right)$, durante 24 horas, de acordo com as RAS (Brasil, 2009). Constatou-se que o lote A apresentou $11,54 \%$; o lote B, 1,69\%; o lote C, $11,90 \%$ e, o lote D, $9,01 \%$. O peso de 100 sementes apresentou o lote A com 19,69 g, o lote B, com 21,58 g, o lote C, com 20,63 g e o lote D com $14,62 \mathrm{~g}$.

No teste padrão de germinação, utilizaram-se 400 sementes de cada lote, somando oito repetições de 50 sementes, distribuídas sobre folha de papel mata-borrão, previamente umedecidas com quantidade de água equivalente a 2,5 vezes o peso do substrato. Os rolos foram acondicionados em câmara de germinação, à temperatura constante de $25^{\circ} \mathrm{C}$. As avaliações foram realizadas aos quinto e oitavo dias após a semeadura, medindo-se o comprimento de radículas e determinando-se a percentagem de sementes germinadas (emissão da radícula primária com $1 \mathrm{~mm}$ ), segundo os critérios estabelecidos pelas RAS (Brasil, 2009).

$\mathrm{O}$ teste de emergência foi realizado em condições ambientes (temperatura média $27,85^{\circ} \mathrm{C}$ ), utilizando-se quatro repetições de 50 sementes, distribuídas em bandejas plásticas, com substrato composto por areia com o teor de água para $100 \%$ da capacidade de retenção. O teste foi avaliado diariamente, contando-se o número de plântulas emergidas, considerando-se, nessa condição, aquelas que continham cotilédones aparentes, acima do nível do substrato. Com esses dados, calculou-se o índice de velocidade de emergência (IVE), seguindo a fórmula proposta por Maguire (1962), e a percentagem de plantas emergidas.

O teste de condutividade elétrica foi realizado com sementes inteiras, excluindo-se as danificadas. Usandose 25 e 50 sementes, depositadas em copos plásticos (200 $\mathrm{mL}$ ), e adicionando-se água deionizada nas quantidades de $75 \mathrm{~mL}$ e $100 \mathrm{~mL}$, por períodos de 2, 4, 8, 12 e 24 horas, à temperatura de $25^{\circ} \mathrm{C}$. Após esses períodos, foi realizada a 
leitura da condutividade elétrica no condutivímetro, sendo os resultados expressos em $\mu \mathrm{S} . \mathrm{cm}^{-1} \cdot \mathrm{g}^{-1}$.

O delineamento experimental utilizado foi o inteiramente casualizado, com quatro repetições, separadamente, para cada lote e teste conduzido, avaliando-se os efeitos de lotes. Os dados foram submetidos à análise de variância, com o auxílio do programa SISVAR (Ferreira, 1999), e, as médias, comparadas pelo teste Tukey a $5 \%$ de probabilidade.

\section{RESULTADOS E DISCUSSÃO}

A percentagem de germinação, primeira contagem, percentagem de emergência e IVE (índice de velocidade de emergência) não apresentaram diferenças significativas entre os lotes (Tabela 1).

Quanto ao parâmetro comprimento de radícula, podese observar que os lotes A e B constituíam-se de sementes de menor vigor enquanto as sementes dos lotes C e D apresentavam-se mais vigorosas.

Os testes de vigor são realizados com objetivo de verificar o potencial de emergência de plântulas, no campo, em condições favoráveis e desfavoráveis (Abrantes et al., 2010). De acordo com Dutra et al. (2007), sementes que apresentam rápido crescimento de radícula podem ser qualificada como potencialmente vigorosas e podem proporcionar maiores taxas de crescimento, no período inicial de estabelecimento da cultura.

Os resultados obtidos pelo teste de condutividade elétrica (Tabela 2) indicaram que todos os tratamentos realizados apresentaram diferenças estatísticas, porém, o que se mostrou mais viável à diferenciação de vigor foi o tratamento das sementes submetidas a 2 horas de embebição, em $100 \mathrm{~mL}$ de água e 25 sementes por repetição, e 2 horas de embebição em $100 \mathrm{~mL}$ de água e 50 sementes por repetição, por serem os mais rápidos e apresentarem os mesmos resultados do teste padrão de germinação.

De maneira geral, a análise dos dados mostrou relação direta com os testes de avaliação da qualidade inicial dos lotes de sementes. Para o feijão-caupi, o teste de condutividade elétrica destacou os lotes $\mathrm{C}$ e D como os de melhor qualidade e os lotes A e B como de pior qualidade, corroborando os dados de comprimento de radícula.
Informações nesse sentido também foram encontradas por Andrade et al. (1995), que verificaram que o teste de condutividade elétrica foi considerado o mais indicado para estimar o vigor de lotes de sementes de cenoura, pela facilidade de execução, rapidez e objetividade.

Com os dados de condutividade elétrica das sementes submetidas a 2 horas de embebição, 25 sementes/ 100 $\mathrm{mL}$ e 50 sementes/ $100 \mathrm{~mL}$ de água, verificou-se que os lotes A e B apresentaram comportamento similar ao dos outros testes aplicados, indicando como sendo os lotes menos vigorosos, já os lotes C e D foram classificados como sendo os de sementes de maior vigor. Os resultados corroboram os obtidos por Dias et al. (1998), que obtiveram sucesso na diferenciação do potencial fisiológico de sementes de feijão-vagem e de quiabo, por meio do teste de condutividade elétrica.

Com relação ao período de embebição das sementes, em suas várias combinações, encontra-se um aumento progressivo das leituras, à medida que se aumentou o período de embebição, corroborando os dados de Dias et al. (1996). Contudo, a liberação foi consideravelmente maior quando as sementes foram submetidas a períodos de 12 e 24 horas, independentemente das combinações de números de sementes e quantidade de água, demonstrando-se menos lixiviadas em período menores. Resultados semelhantes foram encontrados por Gaspar \& Nakagawa, (2002), em milheto, conseguindo distinção entre os lotes com duas horas de embebição. Em sementes de amendoim, Vazolini \& Nakagawa (1999) observaram que o tempo de embebição de três horas foi suficiente para distinguir a qualidade de diferentes lotes.

No teste de condutividade elétrica, o resultado foi semelhante ao do crescimento de radícula, quanto à eficiência de estratificação dos lotes em diferentes níveis de vigor. Maiores valores de lixiviação de solutos resultaram nos menores índices de crescimento radicular, indicando ser este um bom parâmetro de avaliação da qualidade de sementes de feijão-caupi. Segundo Dias \& Marcos Filho (1996), o teste de condutividade elétrica permite identificar lotes com diferentes níveis de vigor, revelando sua importância para um programa de controle de qualidade mais dinâmico e efetivo.

Tabela 1. Dados médios da percentagem de germinação (PG), primeira contagem (PC), percentagem de emergência (PE), índice de velocidade de emergência (IVE) e comprimento de radícula (CR) de sementes de feijão-caupi ${ }^{1}$

\begin{tabular}{lccccc}
\hline Lotes & PG $(\%)$ & PC $(\%)$ & PE $(\%)$ & IVE & CR $(\mathbf{c m})$ \\
\hline A & $99,0^{\mathrm{NS}}$ & $49,5^{\mathrm{NS}}$ & $85,0^{\mathrm{NS}}$ & $11,7^{\mathrm{NS}}$ & $5,8 \mathrm{c}$ \\
$\mathrm{B}$ & $99,7^{\mathrm{NS}}$ & $49,8^{\mathrm{NS}}$ & $85,0^{\mathrm{NS}}$ & $12,2^{\mathrm{NS}}$ & $6,4 \mathrm{c}$ \\
C & $100^{\mathrm{NS}}$ & $50,0^{\mathrm{NS}}$ & $92,5^{\mathrm{NS}}$ & $14,2^{\mathrm{NS}}$ & $8,7 \mathrm{~b}$ \\
D & $97,5^{\mathrm{NS}}$ & $48,7^{\mathrm{NS}}$ & $88,5^{\mathrm{NS}}$ & $15,4^{\mathrm{NS}}$ & $11,7 \mathrm{a}$ \\
\hline CV $(\%)$ & 2,4 & 2,4 & 7,9 & 13,1 & 19,8 \\
\hline
\end{tabular}

${ }^{1}$ Médias seguidas pela mesma letra, na coluna, não diferem entre si pelo teste Tukey a $5 \%$ de probabilidade. ${ }^{\text {Ns }}=$ Não significativo a $5 \%$ de probabilidade.

Rev. Ceres, Viçosa, v. 59, n.4, p. 550-554, jul/ago, 2012 
Tabela 2. Dados médios de condutividade elétrica $\left(\mu \mathrm{S} . \mathrm{cm}^{-1} \cdot \mathrm{g}^{-1}\right)$, utilizando as combinações 25 sementes/ $75 \mathrm{~mL}$ e 25 sementes/ 100 $\mathrm{mL}$ de água e 50 sementes $/ 75 \mathrm{~mL}$ e 50 sementes $/ 100 \mathrm{~mL}$ de água, a $25^{\circ} \mathrm{C}$, de quatro lotes de sementes de feijão-caupi, em cada período de embebição

\begin{tabular}{|c|c|c|c|c|c|}
\hline \multirow{2}{*}{ Lotes } & \multicolumn{5}{|c|}{ Períodos de embebição (h) } \\
\hline & 2 & 4 & 8 & 12 & 24 \\
\hline \multicolumn{6}{|c|}{25 sementes/ $75 \mathrm{~mL}$} \\
\hline A & $60,69 \mathrm{~d}$ & $85,34 \mathrm{c}$ & $97,22 \mathrm{c}$ & $123,46 \mathrm{~d}$ & $151,60 \mathrm{~d}$ \\
\hline B & $50,21 \mathrm{c}$ & $65,92 \mathrm{~b}$ & $68,85 \mathrm{~b}$ & $108,18 \mathrm{c}$ & $125,41 \mathrm{c}$ \\
\hline $\mathrm{C}$ & $34,83 \mathrm{~b}$ & 38,48 a & $45,86 \mathrm{a}$ & $49,21 \mathrm{a}$ & $62,40 \mathrm{a}$ \\
\hline $\mathrm{D}$ & $13,75 \mathrm{a}$ & 39,20 a & 51,01 a & $72,66 \mathrm{~b}$ & 84,98 b \\
\hline \multicolumn{6}{|c|}{ C.V. $(\%)=7,92$} \\
\hline \multicolumn{6}{|c|}{25 sementes/ $100 \mathrm{~mL}$} \\
\hline A & $45,54 \mathrm{c}$ & $61,75 \mathrm{c}$ & $80,65 \mathrm{~b}$ & $92,25 \mathrm{~d}$ & $122,11 \mathrm{~d}$ \\
\hline B & $37,77 \mathrm{c}$ & $49,38 \mathrm{~b}$ & $81,29 \mathrm{~b}$ & $81,29 \mathrm{c}$ & $106,40 \mathrm{c}$ \\
\hline $\mathrm{C}$ & $22,51 \mathrm{~b}$ & $28,31 \mathrm{a}$ & $32,19 \mathrm{a}$ & $35,88 \mathrm{a}$ & $42,99 \mathrm{a}$ \\
\hline D & $8,94 \mathrm{a}$ & 29,07 a & $40,25 \mathrm{a}$ & $62,68 \mathrm{~b}$ & $59,43 \mathrm{~b}$ \\
\hline \multicolumn{6}{|c|}{ C.V. $(\%)=7,92$} \\
\hline \multicolumn{6}{|c|}{50 sementes/ 75 mL } \\
\hline A & $59,99 \mathrm{~d}$ & $77,50 \mathrm{c}$ & $98,27 \mathrm{c}$ & $113,26 \mathrm{c}$ & $158,52 \mathrm{~d}$ \\
\hline B & $48,64 \mathrm{c}$ & $64,81 \mathrm{~b}$ & $67,72 \mathrm{~b}$ & $110,38 \mathrm{c}$ & $103,03 \mathrm{c}$ \\
\hline $\mathrm{C}$ & $35,00 \mathrm{~b}$ & $38,93 \mathrm{a}$ & $44,21 \mathrm{a}$ & $53,16 \mathrm{a}$ & $56,23 \mathrm{a}$ \\
\hline D & $12,26 \mathrm{a}$ & $37,40 \mathrm{a}$ & 49,19 a & $66,93 \mathrm{~b}$ & $67,59 \mathrm{~b}$ \\
\hline \multicolumn{6}{|c|}{ C.V. $(\%)=7,92$} \\
\hline \multicolumn{6}{|c|}{50 sementes/ $100 \mathrm{~mL}$} \\
\hline A & $45,55 \mathrm{c}$ & $59,11 \mathrm{c}$ & $71,82 \mathrm{c}$ & $91,61 \mathrm{c}$ & $113,22 \mathrm{~d}$ \\
\hline B & $39,22 \mathrm{c}$ & $47,21 \mathrm{~b}$ & $49,73 \mathrm{~b}$ & $74,53 \mathrm{~b}$ & $92,13 \mathrm{c}$ \\
\hline $\mathrm{C}$ & $25,67 \mathrm{~b}$ & $28,66 \mathrm{a}$ & $32,04 \mathrm{a}$ & $39,07 \mathrm{a}$ & $41,76 \mathrm{a}$ \\
\hline D & $9,94 \mathrm{a}$ & $28,34 \mathrm{a}$ & $31,86 \mathrm{a}$ & $42,38 \mathrm{a}$ & $55,45 \mathrm{~b}$ \\
\hline
\end{tabular}

C.V. $(\%)=7,92$

As médias seguidas pela mesma letra, na coluna, não diferem entre si pelo teste de Tukey a 5\% de probabilidade. ${ }^{\text {NS }}=$ Não significativo a $5 \%$ de probabilidade.

Miranda et al. (2001), trabalhando com sementes de sorgo, observaram que os testes de vigor aplicados às sementes, quando comparados com os testes de germinação, proporcionaram informações mais detalhadas sobre níveis de qualidade dos diferentes lotes de sementes, principalmente o teste de condutividade elétrica. Portanto, destaca-se a importância da utilização conjunta dos resultados de vários testes para a avaliação do potencial fisiológico de sementes, conforme considerou Marcos Filho (1999b).

\section{CONCLUSÕES}

O teste de condutividade elétrica é eficiente na separação dos lotes de feijão-caupi, com relação ao potencial fisiológico, sendo a condição mais adequada para a realização desse teste a utilização de embebição de duas horas, com $100 \mathrm{~mL}$ de água e 25 sementes, por repetição; ou duas horas, com $100 \mathrm{~mL}$ de água e 50 sementes, por repetição.

\section{REFERÊNCIAS}

Abrantes FL, Kulczynski SM, Soratto RP \& Barbosa MMM (2010) Nitrogênio em cobertura e qualidade fisiológica e sanitária de sementes de painço (Panicum miliaceum L.). Revista Brasileira de Sementes, 32:106-115.

Andrade RN, Santos DSB, Santos Filho BG \& Mello VDC (1995) Correlação entre testes de vigor em sementes de cenoura armazenadas por diferentes períodos. Pesquisa Agropecuária Gaúcha, $1: 153-162$.

AOSA - Association of official seed analysts (1983) Seed vigor testing handbook. Lincoln, NE, USA, 93p. (Contribution, 32).

AOSA - Association of official seed analysts (2002) Seed vigor testing handbook. Lincoln, NE, USA, 105p. (Contribution, 32).

Brasil (2009) Regras para análise de sementes. Brasília, Ministério da Agricultura, Pecuária e Abastecimento, Mapa/ACS. 399p.

Carvalho NM de \& Nakagawa J (2000) Sementes: Ciência, tecnologia e produção. $4^{\mathrm{a}}$ ed. Jaboticabal, Funep. 588p.

Carvalho MLM, França Neto JB \& Krzyzanowski FC (2006) Controle e qualidade na produção de sementes. Informe Agropecuário, 27:52-58.

Dias DCFS \& Marcos Filho J (1996) Testes de condutividade elétrica para avaliação do vigor de sementes de soja (Glycine max (L.) Merrill). Scientia Agrícola, 53:31-42.

Rev. Ceres, Viçosa, v. 59, n.4, p. 550-554, jul/ago, 2012 
Dias DCFS, Vieira NA \& Bhéring MC (1998) Condutividade elétrica e lixiviação de potássio para avaliação do vigor de sementes de hortaliças: feijão-de-vagem e quiabo. Revista Brasileira de Sementes, 20:408-413.

Dias DCFS, Vieira NA \& Bhéring MC (1996) Estudo dos testes de condutividade elétrica e lixiviação de potássio para avaliação do vigor de sementes de hortaliças. I. Couve-flor, cebola e cenoura. In: $15^{\circ}$ Seminario Panamericano de Semillas, Gramado. Anais, CESM, FELAS. p.28.

Dutra AS \& Teófilo EM (2007) Envelhecimento acelerado para avaliar o vigor de sementes de feijão-caupi. Revista Brasileira de Sementes, 29:193-197.

Dutra AS, Teófilo EM \& Medeiros Filho S (2007) Qualidade fisiológica de sementes de feijão-caupi em quatro regiões do estado do Ceará. Revista Brasileira de Sementes, 29:11-116.

Ferreira DF (1999) Sistema para análise de variância para dados balanceados (SISVAR). Lavras, UFLA. 92p.

Gaspar CM \& Nakagawa J (2002) Teste de condutividade elétrica em sementes de milheto. Revista Brasileira de Sementes, 24:8289.

Maguire JD (1962) Speed of germination-aid selection and evaluation for seedling emergence and vigor. Crop Science, 2:176-177.
Marcos Filho J (1986) Germinação de sementes. In: $1^{a}$ Semana de atualização em produção de sementes, Piracicaba, Fundação Cargill. p.11-39.

Marcos Filho J (1999a) Testes de vigor: Importância e utilização. In: Krzyzanowski FC, Vieira RD \& França Neto JB (Eds.) Vigor de sementes: Conceitos e testes. Londrina, ABRATES. p.1-21.

Marcos Filho J (1999b) Teste de envelhecimento acelerado. In: Krzyzanowski FC, Vieira RD \& França Neto JB (Eds.) Vigor de sementes: Conceitos e testes. Londrina, ABRATES. p.1-24.

Mendonça EAF, Ramos NP \& Fessel SA (2003) Adequação da metodologia do teste de deterioração controlada para sementes de brócolis (Brassica oleracea L. - var. Itálica). Revista Brasileira de Sementes, 25:18-24.

Miranda DM, Novembre ADLC \& Chamma HMCP (2001) Avaliação do potencial fisiológico de sementes de sorgo pelo teste de envelhecimento acelerado. Revista Brasileira de Sementes, 23:226-231.

Vazolini S \& Nakagawa J (1999) Teste de condutividade elétrica em sementes de amendoim: efeito de teor de água e de período de embebição. Revista Brasileira de Sementes, 21:46-52.

Vieira RD \& Krzyzanowski FC (1999) Teste de condutividade elétrica. In: Krzyzanowski FC, Vieira RD \& França Neto JB (Eds.) Vigor de sementes: Conceitos e testes. Londrina, ABRATES. p.1-26. 